\title{
Astrocytes Impose Postburst Depression of Release Probability at Hippocampal Glutamate Synapses
}

\author{
My Andersson and Eric Hanse \\ Institute of Neuroscience and Physiology, Gothenburg University, 40530 Göteborg, Sweden
}

\begin{abstract}
Many neurons typically fire action potentials in brief, high-frequency bursts with specific consequences for their synaptic output. Here we have examined short-term plasticity engaged during burst activation using electrophysiological recordings in acute rat hippocampal slices. We show that CA3-CA1 glutamate synapses exhibit a prominent depression of presynaptic release probability for $\sim 1 \mathrm{~s}$ after such a burst. This postburst depression exhibits a distinct cooperativity threshold, is abolished by inhibiting astrocyte metabolism and astrocyte calcium signaling, and is not operational in the developing hippocampus. Our results suggest that astrocytes are actively involved in short-term synaptic depression, shaping synaptic activity during behaviorally relevant neural activity.
\end{abstract}

\section{Introduction}

Synaptic transmission is highly frequency dependent, mainly because the probability of releasing transmitter depends critically on the recent (milliseconds to seconds) pattern of action potentials (Schneggenburger et al., 2002; Zucker and Regehr, 2002). Synapses with low basal release probability typically exhibit facilitation and augmentation, whereas high-release-probability synapses exhibit depression in response to repetitive synaptic activation (Abbott and Regehr, 2004). This short-term synaptic plasticity relies on both release-independent and release-dependent mechanisms. Independent of release, the calcium influx during the action potential contributes to residual calcium in the terminal, which is involved in mediating facilitation, augmentation, and depression (Neher and Sakaba, 2008). Release-dependent shortterm synaptic depression may occur either because of depletion of vesicles or because released neurotransmitter feeds back onto presynaptic autoreceptors to negatively modulate release probability. At cortical glutamate synapses, very little is known about the contribution to short-term depression of such homosynaptic negative feedback. In the present work, we show that brief bursts of action potentials are efficient triggers of homosynaptic negative feedback at hippocampal glutamate synapses. Surprisingly, we found that this homosynaptic negative feedback requires astrocyte activation, thus indicating that glutamate released from the presynaptic terminal acting on presynaptic autoreceptors is not sufficient.

Astrocyte processes closely enwrap the presynaptic and postsynaptic elements of many cortical glutamate synapses (Witcher et al., 2007), making tripartite synapses (Araque et al., 1999). By responding to presynaptically released glutamate and by releasing gliotrans-

Received Aug. 12, 2009; revised March 7, 2010; accepted March 21, 2010.

This work was supported by grants from the Swedish Research Council (project number 12600) and by the Sahlgrenska Hospital (Agreement concerning Research and Education of Doctors). We thank Dr. Fredrik Asztely for helpful comments on this manuscript.

Correspondence should be addressed to My Andersson, Institute of Neuroscience and Physiology, Gothenburg University, Box 432, Medicinaregatan 11, 40530 Göteborg, Sweden. E-mail: my.andersson@physiol.gu.se.

DOI:10.1523/JNEUROSCI.3957-09.2010

Copyright $\odot 2010$ the authors $\quad 0270-6474 / 10 / 305776-05 \$ 15.00 / 0$ mitters, the astrocyte part of the tripartite synapse is strategically positioned to modulate release probability in an activity-dependent manner (Newman, 2003). Indeed, several studies have shown that gliotransmitters can modulate release probability at cortical synapses (Robitaille, 1998; Serrano et al., 2006; Zhang et al., 2003; Liu et al., 2004; Pascual et al., 2005; Andersson et al., 2007; Jourdain et al., 2007), and we now show that astrocytes play an active role in mediating homosynaptic short-term synaptic depression.

\section{Materials and Methods}

Slice preparation and solutions. Experiments were performed on hippocampal slices from 6- to 52-d-old Wistar rats. The animals were killed in accordance with the guidelines of the local ethical committee for animal research. Rats were anesthetized with isoflurane (Abbott) before decapitation. The brain was removed and placed in an ice-cold solution containing the following (in $\mathrm{mm}$ ): 140 cholineCl, $2.5 \mathrm{KCl}, 0.5 \mathrm{CaCl}_{2}, 7$ $\mathrm{MgCl}_{2}, 25 \mathrm{NaHCO}_{3}, 1.25 \mathrm{NaH}_{2} \mathrm{PO}_{4}, 1.3$ ascorbic acid, and 7 dextrose. Transverse hippocampal slices (300-400 $\mu \mathrm{m}$ thick) were cut with a vibratome (HM 650V Microm) in the same ice-cold solution, and they were subsequently stored in artificial CSF (ACSF) containing the following (in mM): $124 \mathrm{NaCl}, 3 \mathrm{KCl}, 2 \mathrm{CaCl}_{2}, 4 \mathrm{MgCl}_{2}, 26 \mathrm{NaHCO}_{3}, 1.25$ $\mathrm{NaH}_{2} \mathrm{PO}_{4}, 0.5$ ascorbic acid, 3 myo-inositol, 4 D,L-lactic acid, and 10 D-glucose. After at least $1 \mathrm{~h}$ of storage at $25^{\circ} \mathrm{C}$, a single slice was transferred to a recording chamber where it was kept submerged in a constant flow $\left(\sim 2 \mathrm{ml} \cdot \min ^{-1}\right)$ at $30-32^{\circ} \mathrm{C}$. The perfusion fluid contained (in mм) $124 \mathrm{NaCl}, 3 \mathrm{KCl}, 4 \mathrm{CaCl}_{2}, 4 \mathrm{MgCl}_{2}, 26 \mathrm{NaHCO}_{3}, 1.25 \mathrm{NaH}_{2} \mathrm{PO}_{4}$, and $10 \mathrm{D}$-glucose. Picrotoxin $(100 \mu \mathrm{M})$ and D-AP5 (50 $\mu \mathrm{M}$, except for when recording NMDA currents) was present in the perfusion fluid to block $\mathrm{GABA}_{\mathrm{A}}$ and NMDA receptor-mediated activity, respectively. All solutions were continuously bubbled with $95 \% \mathrm{O}_{2}$ and $5 \% \mathrm{CO}_{2}(\mathrm{pH}$ 7.4). The higher than normal $\mathrm{Ca}^{2+}$ and $\mathrm{Mg}^{2+}$ concentrations were used to inhibit network activity.

Field recordings and analysis. Electrical stimulation of Schaffer collateral/commissural axons and recordings of synaptic responses were carried out in the stratum radiatum of the CA1 region. Stimuli consisted of biphasic constant-current pulses (15-80 $\mu \mathrm{A}, 200 \mu \mathrm{s}$, STG 1002 Multi Channel Systems MCS) delivered through tungsten wires (resistance $\sim 0.1 \mathrm{M} \Omega$ ). One stimulation electrode was positioned in the stratum radiatum with a distance of $100 \mu \mathrm{m}$ from the recording electrode. The synaptic input was activated every $10 \mathrm{~s}$, and stimulation intensity was 
A
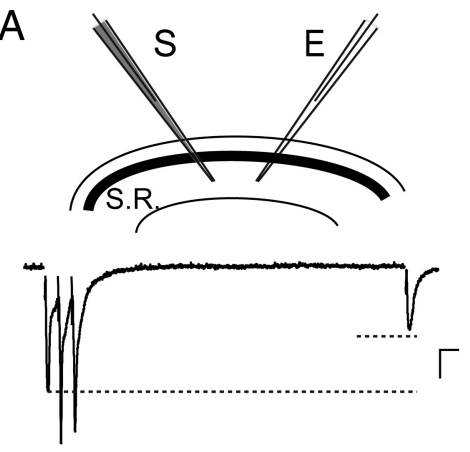

C

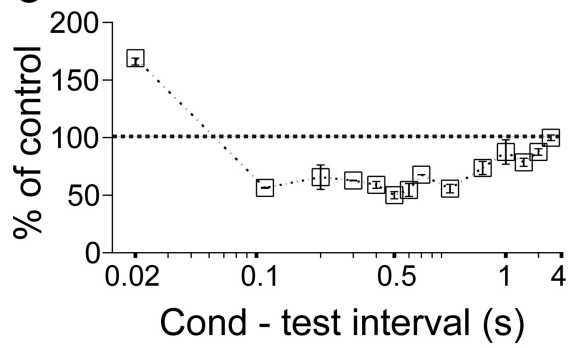

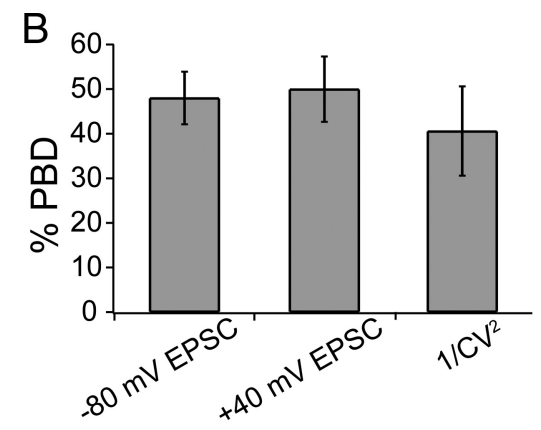

D

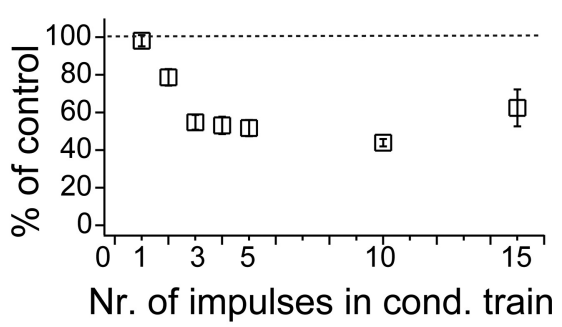

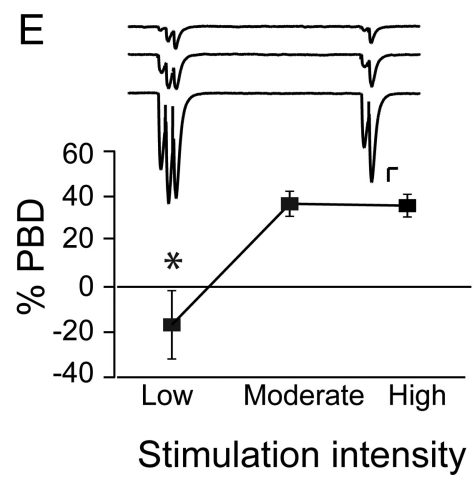

Figure 1. A brief conditioning synaptic burst elicits PBD at hippocampal CA3-CA1 synapses. A, Field EPSP recordings illustrating the PBD elicited by a three-impulse, $50 \mathrm{~Hz}$ conditioning burst $500 \mathrm{~ms}$ before the test stimulation. Calibration: $20 \mathrm{~ms}, 100 \mu \mathrm{V}$. Above is a schematic illustration of the placement of stimulation $(S)$ and extracellular recording electrode (E) in the CA1 stratum radiatum (S.R.). B, Comparison of PBD recorded using AMPA EPSCs $(n=7)$ and NMDA EPSCs $(n=7)$ as well as the decrease of $1 / \mathrm{CV}^{2}$ for the AMPA EPSCS $(n=5)$. C, The PBD is maximal at conditioning (Cond)-test intervals of $0.1-1 \mathrm{~s}$ (conditioning burst consisted of 3 impulses at $50 \mathrm{~Hz}, n=4-8$ per bar). $\boldsymbol{D}$, Increasing the number ( $\mathrm{Nr}$.) of stimuli in the conditioning burst beyond three at $50 \mathrm{~Hz}$ does not increase the magnitude of the PBD. The conditioning-test interval was $500 \mathrm{~ms}(n=4-7$ per bar). $\boldsymbol{E}$, Whole-cell recordings of AMPA EPSCs reveal a cooperativity threshold for the PBD. The top traces illustrate a conditioning-test sequence using AMPA EPSCS evoked using very low, moderate, and high stimulation intensities. Calibration: $20 \mathrm{~ms}, 50 \mathrm{pA}$. The bottom graph summarizes five experiments the same as that illustrated on top $\left({ }^{*} p<0.05\right)$.

[except in the whole-cell experiments examining the cooperativity requirement (Fig. 1E)] adjusted such that spike activity was observed on the second and/or third fEPSP in the three impulse conditioning burst. Field EPSPs were recorded with a glass micropipette (filled with perfusion fluid or $1 \mathrm{M} \mathrm{NaCl}$, resistance 1-2 $\mathrm{M} \Omega$ ). Field EPSPs were sampled at $10 \mathrm{kHz}$ with an EPC-9 amplifier (HEKA Elektronik) and filtered at $1 \mathrm{kHz}$. Evoked responses were analyzed offline using custom-made IGOR Pro (WaveMetrics) software. Field EPSP magnitude was estimated by linear regression over the first $0.8 \mathrm{~ms}$ of the initial slope. The presynaptic volley was measured as the slope of the initial positive-negative deflection, and it was not allowed to change by $>10 \%$ during the experiment.

Patch-clamp recordings of pyramidal cells and astrocytes. Pyramidal cells were visually identified in the CA1 area with IR-DIC video microscopy (Nikon) and patched with an intracellular solution containing the following (in mM): 120 Cs-methane sulfonate, $2 \mathrm{NaCl}, 10$ HEPES, 5 QX314, 4 Mg-ATP, 0.4 GTP, and 20 BAPTA, pH 7.2. For measuring AMPA and NMDA EPSCs, the cell was held at $-80 \mathrm{mV}$ and $+40 \mathrm{mV}$, respectively. Ten micromolar NBQX was present in the extracellular solution to block AMPA EPSCs when recording NMDA EPSCs. Evoked responses were analyzed offline using IGOR Pro (WaveMetrics) software. Amplitudes were measured on average-sweeps of 18 consecutive sweeps. Astrocytes in the stratum radiatum were identified by their small soma and, when patched, by their passive electrophysiological properties (Zhou et al., 2006). The intracellular solution contained the following (in $\mathrm{mM}$ ): $120 \mathrm{KCl}, 2$ $\mathrm{NaCl}, 20$ HEPES, $4 \mathrm{Mg}$-ATP, $0.4 \mathrm{GTP}$, and 50 BAPTA or $120 \mathrm{KCl}, 2 \mathrm{NaCl}, 20$ HEPES, $4 \mathrm{Mg}$ ATP, 0.4 GTP, and $10 \mathrm{~mm}$ BAPTA. Patch pipettes $(1.5 \mathrm{~mm} / 0.86 \mathrm{~mm}$; borosilicate, Clark Electrochemical Instruments) were pulled with a horizontal puller (Sutter Instruments) to a resistance of 3-6 M $\Omega$. Series resistance was measured using a $5 \mathrm{~ms}, 10 \mathrm{mV}$ hyperpolarizing pulse and was not allowed to change by $>15 \%$ during the experiment.

Data are expressed as means \pm SEM. Statistical significance for paired and independent samples was evaluated using Student's $t$ test.

Drugs. Picrotoxin and fluoroacetate (FAc) came from Sigma-Aldrich, D-AP5 and NBQX from Accent Scientific, and endothelin-1 from Neosystems.

\section{Results}

Hippocampal CA3-CA1 glutamatergic synapses, as a population, exhibit facilitation immediately (tens of milliseconds) after short synaptic burst activation, and augmentation is observed during seconds after longer synaptic bursts (Andersen, 1960; Gustafsson et al., 1989). However, at intermediate intervals $(0.1-1 \mathrm{~s})$, burst activation results in depression as illustrated in Figure 1. Even a single conditioning burst consisting of three impulses at $50 \mathrm{~Hz}$ gives rise to a prominent synaptic depression of $41 \pm 3 \%$ (compared to the first fEPSP in the conditioning burst, $n=28$, $p<0.0001$ ) $0.5 \mathrm{~s}$ after the burst (Fig. $1 A$ ). This postburst depression (PBD) of the fEPSP was associated with an increase in the paired-pulse ratio of $14 \pm 5 \%(n=28$, $p=0.004)$, and with no change of the presynaptic volley $(3 \pm 1 \%, n=28, p=$ 0.98). The PBD was observed to the same extent using whole-cell recordings from CA1 pyramidal neurons, both when reported using AMPA EPSCs at $-80 \mathrm{mV}$ and when reported using NMDA EPSCs recorded at $+40 \mathrm{mV}$ (Fig. $1 B$ ). The NMDA EPSCs were recorded in the presence of $10 \mu \mathrm{M}$ NBQX, an AMPA/kainate antagonist, implying that AMPA and kainate receptors are not necessary for the PBD. The PBD of the AMPA EPSC was associated with a comparable decrease in $1 / \mathrm{CV}^{2}$ (Fig. $1 B$ ). Twenty millimolar BAPTA was included in the pipette solution in these whole-cell recording experiments, indicating that a rise in postsynaptic calcium is not necessary for the generation of the PBD. Based on the equal depression of AMPA and NMDA EP$\mathrm{SCs}$, the increased paired-pulse ratio, and the decreased $1 / \mathrm{CV}^{2}$ ratio, we conclude that the PBD is expressed as a depression in release probability. 
The PBD was most evidently expressed when there was a delay between conditioning burst and the test stimulus of 0.1-1 s (Fig. $1 C)$. Changing the intraburst frequency in the conditioning burst indicated that frequencies higher than $20-30 \mathrm{~Hz}$ produced maximal PBD (supplemental Fig. 1, available at www.jneurosci.org as supplemental material). Moreover, changing the number of impulses in the conditioning burst showed that, whereas a single stimulus is insufficient, three impulses at $50 \mathrm{~Hz}$ produced maximal PBD (Fig. 1D). These findings indicate that the PBD is saturable and that there is a certain threshold for the induction. It is noteworthy that the conditioning-pause-test protocol resulted in larger depression than did continuous high-frequency activation during the same period. Thus, the 20th fEPSP in a 20 impulse, $50 \mathrm{~Hz}$ train was only depressed by $16 \pm 10 \%$ (of the first fEPSP, $n=5$ ). Together, these results would indicate that the $\mathrm{PBD}$ is particularly engaged during theta burst activity. Especially during exploratory behavior and during REM sleep, hippocampal pyramidal neurons typically fire action potentials in short high-frequency $(40-100 \mathrm{~Hz}$ ) bursts with interburst intervals corresponding to the theta frequency $(4-10 \mathrm{~Hz}$ ) (Lisman and Buzsáki, 2008). This theta burst activity is thought to be critical for coding information in the hippocampus (Lisman and Buzsáki, 2008), and it is an efficient mean to induce long-term synaptic plasticity (Larson et al., 1986; Huerta and Lisman, 1996).

In contrast to most other forms of short-term plasticity (Zucker and Regehr, 2002), it was necessary to simultaneously activate a certain minimal number of synapses to induce the PBD. As shown in Figure $1 E$, a stimulation intensity low enough to only activate a few synapses targeting the CA1 pyramidal neuron recorded from (as indicated by frequent synaptic failures) was not sufficient to induce the PBD. Recruiting more synapses, by increasing the stimulation intensity, revealed a PBD, which did not become larger by further synaptic recruitment, showing that the PBD exhibits a distinct cooperativity threshold. This cooperativity threshold for the PBD contrasts with the lack of such requirement for paired-pulse plasticity, which remained the same at the three different stimulation intensities $(1.46 \pm 0.04,1.47 \pm$ $0.03,1.47 \pm 0.03, n=5)$. The cooperativity threshold for the PBD is, however, likely low, since stimulation intensities evoking fEPSPs up to 10 times smaller than fEPSPs showing signs of population spike activity were still sufficient to elicit full $\operatorname{PBD}(n=5)$. Since the PBD was unaffected by voltage clamping the membrane potential (at $-80 \mathrm{mV}$ and at $+40 \mathrm{mV}$ ) and by calcium buffering in the postsynaptic neuron, it is unlikely that the postsynaptic neurons are responsible for mediating the cooperativity effect. Two other possibilities, which could account for the cooperativity effect, are spillover of neurotransmitter (Arnth-Jensen et al., 2002) and activity-induced release of gliotransmitters from astrocytes.

Since astrocyte function and morphology develop during an extended postnatal period (Konietzko and Muller, 1994; Bushong et al., 2004; Schools et al., 2006; Andersson et al., 2007), activityinduced release of gliotransmitters from astrocytes would be expected to increase with development. Spillover, on the other hand, would rather be expected to be favored early in development when astrocyte processes around synapses and glutamate uptake are poorly developed (Piet et al., 2004; Diamond, 2005). The developmental profile of the PBD (Fig. $2 A$ ), emerging at postnatal day 10 and being fully developed at postnatal day 20 , would thus be more consistent with activity-induced release of gliotransmitters from astrocytes than with spillover. To more directly test whether the $\mathrm{PBD}$ required some form of astrocyte signaling, we elicited the PBD before and after the application of the glial metabolic inhibitor FAc (Fonnum et al., 1997; Andersson et al., 2007).
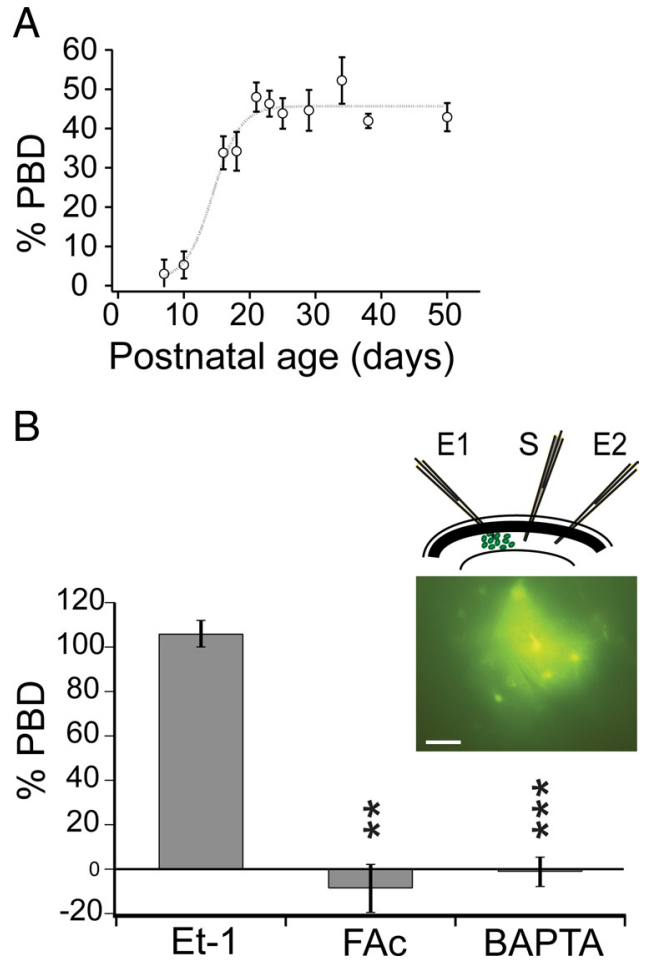

Figure 2. Developmental profile and astrocyte involvement in the PBD. $A$, Developmental profile of the PBD. Results from rats of different postnatal days were binned into 11 groups ( $n=$ 5-15 per group), and a sigmoid curve was fit to the data points. $\boldsymbol{B}$, The PBD is blocked by the glial metabolic inhibitor FAc ( $10 \mathrm{~mm}$ FAc) and by BAPTA ( $50 \mathrm{~mm}$ ) infused into the gap junctioncoupled astrocyte network, but not by the gap junction blocker endothelin-1 (Et-1, $1 \mu \mathrm{m})$. The control PBD before FAc was $42 \pm 5 \%(n=11)$ and was reduced to $-9 \pm 6 \%\left({ }^{* *} p<0.005\right)$ after FAc. The control PBD before BAPTA ( $43 \pm 3 \%, n=5)$ was recorded from a region in the slice without BAPTA-filled astrocytes and was compared with the $P B D$ recorded from a region in the same slice with BAPTA-filled astrocytes $\left(-4 \pm 3 \%, n=5,{ }^{* * *} p<0.001\right)$, as schematically indicated on top (scale bar indicates $50 \mu \mathrm{m}$ ). S, Stimulating electrode; E1, E2, extracellular recording electrodes.

Consistent with a critical role for astrocyte signaling, FAc completely blocked the PBD (Fig. $2 B$ ). We have previously shown that endothelin-1 closes astrocyte gap junctions and inhibits an astrocyte-mediated transient heterosynaptic depression (Blomstrand et al., 2004; Andersson et al., 2007). However, endothelin-1 did not affect the magnitude of the PBD (Fig. 2 B), indicating that signaling through astrocyte gap junction is not required for this homosynaptic form of astrocyte-mediated synaptic depression.

To test whether a rise in astrocyte calcium is a necessary step in the generation of $\mathrm{PBD}$, we patched gap junction-coupled astrocytes in the stratum radiatum with a pipette solution containing the calcium chelator BAPTA and Lucifer yellow. To allow diffusion of BAPTA to a large number of gap junction-coupled astrocytes in 20 min (Serrano et al., 2006), we used a high concentration of BAPTA (50 mM). The diffusion of Lucifer yellow was used to estimate the diffusion of the calcium chelator in the astrocytic network as Lucifer yellow is of comparable molecular size to BAPTA (Lucifer yellow MW: 457 to BAPTA MW: 476). Both of these are well below the $1 \mathrm{kDa}$ size limit for gap junction pores (Giaume et al., 2010). When Lucifer yellow and BAPTA had diffused to gap junction-coupled astrocytes for $20 \mathrm{~min}$, the patch pipette was withdrawn and an extracellular recording pipette was placed in close vicinity (within $20 \mu \mathrm{m}$ ) to the originally patched astrocyte. Under these conditions, the PBD could not be elicited (Fig. 2 B), although normal PBD was elicited when recorded from another 

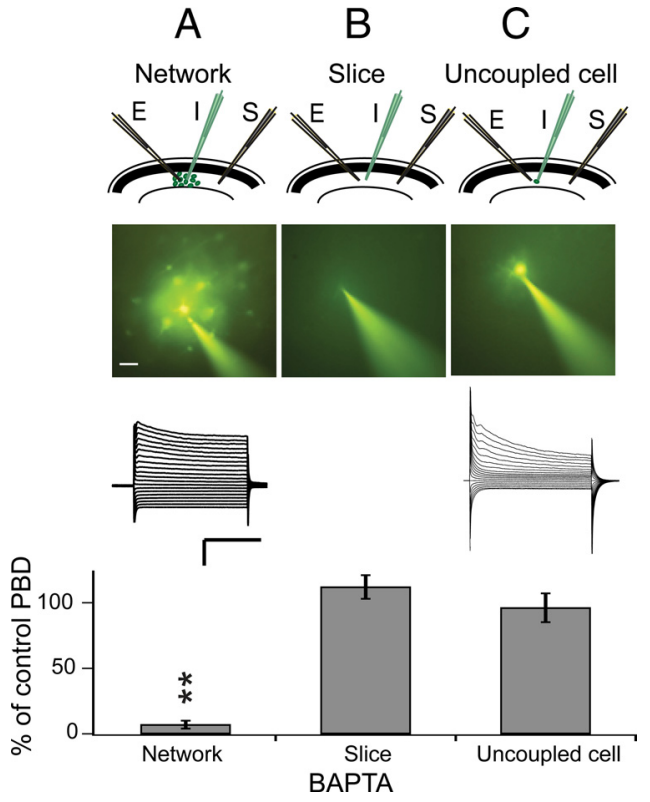

Figure 3. Astrocyte calcium signaling is critically involved in the generation of the PBD. The effect on the PBD is evaluated as relative change of control PBD in three different situations: "Network," "Slice," and "Uncoupled cell." $A$, "Network":BAPTA (50mM) and Luciferyellow were infused for $20 \mathrm{~min}$ via the patch pipette into a gap junction-coupled astrocyte exhibiting passive electrophysiological properties (scale bar indicates $20 \mu \mathrm{m})$. Control PBD before BAPTA infusion ( $45 \pm 5 \%, n=5$ ) was significantly different from the PBD 20 min after BAPTA infusion ( $\left.{ }^{* *} p<0.005\right)$. B, "Slice": a BAPTAcontaining patch pipette was simply placed into the slice for $20 \mathrm{~min}$. Control PBD before placement of BAPTA-containing pipette in the slice ( $41 \pm 7 \%, n=5$ ) was not significantly different from the PBD 20 min after $(p>0.05)$. C, "Uncoupled cell": BAPTA infused for 20 min via the patch pipette into an uncoupled cell with nonpassive electrophysiological properties. Control PBD before patching ( $47 \pm$ $4 \%, n=3$ ) was not significantly different from the PBD 20 min after patching ( $p>0.05$ ). Electrophysiological properties were tested using voltage steps of $10 \mathrm{mV}$ from $-100 \mathrm{mV}$ to $+50 \mathrm{mV}$ (calibration: $50 \mathrm{~ms}, 100 \mathrm{pA}$ ). Top parts of the panels show schematic drawings depicting placement of extracellular recording electrode (E), intracellular recording electrode (I), and stimulation electrode (S) in the three different situations.

region without BAPTA/Lucifer yellow-filled astrocytes in the CA1 str. radiatum of the same slice. A lower concentration of BAPTA (10 mM) allowed to diffuse for $20 \mathrm{~min}$ did not fully block, but substantially inhibited the PBD to $27 \pm 9 \%$ of control $(n=3$, $p=0.008)$. Notably, paired-pulse plasticity was unaffected by the presence of a high concentration of BAPTA in the astrocytes $(102 \pm 5 \%, n=5, p=0.6)$. Experiments using the inhibitor of the sarcoplasmic reticulum calcium pump, cyclopiazonic acid $(10 \mu \mathrm{M})$, indicated that release of calcium from internal stores is involved in the generation of the PBD. In the presence of cyclopiazonic acid, the PBD was decreased to $63 \pm 12 \%$ of control $(n=5, p=0.036)$.

The blockade of PBD by chelating astrocyte calcium was also observed in another set of experiments in which we first recorded a control PBD and then patched an astrocyte in the vicinity of the extracellular recording pipette with a solution containing $50 \mathrm{~mm}$ BAPTA and Lucifer yellow. Twenty minutes after having patched the astrocyte, we again applied the PBD protocol. As illustrated in Figure $3 A$, the PBD was completely blocked by filling the astrocyte network with $50 \mathrm{~mm}$ BAPTA. As a control, we repeated the experiment without patching an astrocyte, simply positioning the patch pipette into the slice for $20 \mathrm{~min}$. In these control experiments, the PBD was unaffected (Fig. 3B). Furthermore, in three experiments we patched uncoupled astrocytes with nonpassive electrophysiological properties. Patching such astrocytes did not affect the PBD (Fig. 3C).
To examine whether continuous train stimulation, as opposed to burst stimulation, elicits this astrocyte-mediated depression, we examined the responses to trains of 20 impulses at 0.2 $\mathrm{Hz}, 2 \mathrm{~Hz}$, and $50 \mathrm{~Hz}$, in the region of the slice in which $50 \mathrm{~mm}$ BAPTA was infused into a gap junction-coupled astrocyte, as well as in a control region of the same slice (compare Fig. 2C). Despite inhibiting the $\mathrm{PBD}$, these trains were largely unaffected by strongly buffering intracellular astrocyte calcium (supplemental Fig. 2, available at www.jneurosci.org as supplemental material).

\section{Discussion}

We have identified a form of short-term synaptic plasticity, a $\mathrm{PBD}$, which critically depends on astrocyte calcium signaling. It is expressed as a reduction in release probability and becomes fully operational after the third postnatal week. A brief high-frequency burst is sufficient to induce this form of synaptic negative feedback, whose expression also involves neighboring inactive synapses (Andersson et al., 2007).

An active, signaling role of astrocytes has previously been implicated for different forms of long-term synaptic plasticities in the hippocampus (Pascual et al., 2005; Serrano et al., 2006; Henneberger et al., 2010). Astrocyte release of ATP/adenosine has been linked to heterosynaptic long-term depression (Pascual et al., 2005; Serrano et al., 2006), and release of D-serine has been linked to the induction of NMDA receptor-dependent long-term potentiation (Henneberger et al., 2010). The induction of these forms of synaptic plasticity appears to require more extensive synaptic activation than what is required for the induction of the PBD. An interesting possibility is that astrocytes can decode different patterns of synaptic activity into different modes of calcium signaling (Verkhratsky et al., 1998; Perea et al., 2009; Santello and Volterra, 2009) and into release of different constellations of gliotransmitters (Duan et al., 2003; Bowser and Khakh, 2007; Zhang et al., 2007; Li et al., 2008; Marchaland et al., 2008). The type of calcium signaling as well as the critical gliotransmitters involved in the generation of $\mathrm{PBD}$ remains, however, to be determined.

The presently described PBD resembles the negative synapseglia-synapse feedback loop that has previously been described in the peripheral nervous system (Robitaille, 1998; Perez-Gonzalez et al., 2008). A peculiar feature of the PBD is that it is most evidently expressed during a restricted time window (0.1-1 s) after, but not during, high-frequency activity. A similar phenomenon has been shown at the calyx of Held synapse, where activation of metabotropic glutamate autoreceptors did not affect the response during continuous high-frequency activation, but slowed down the recovery from postburst depression (Billups et al., 2005). The reasons why continuous repetitive activation is ineffective in generating the astrocyte-dependent depression will require further studies. It is, however, likely that continuous activation at the lower frequencies $(0.2$ and $2 \mathrm{~Hz})$ is below threshold for sufficient activation of the astrocytes (cf. supplemental Fig. 1, available at www.jneurosci.org as supplemental material). The reason why astrocyte-dependent depression is not evident during continuous activation at the higher frequency $(50 \mathrm{~Hz})$ might rather relate to the specific mode of presynaptic release under this condition. During high-frequency activation release probability is limited by the recruitment of release-ready vesicles, rather than being limited by the number of release-ready vesicles and their probability of release (Sakaba et al., 2002; Abrahamsson et al., 2005).

It is notable that the PBD is induced in almost an all-or-none fashion. A certain number of synapses need to cooperate, and 
PBD is induced by only three impulses at high frequency. Since hippocampal pyramidal neurons typically fire action potentials in short bursts in the behaving animal (Lisman and Buzsáki, 2008), this relatively modest threshold would suggest that the $\mathrm{PBD}$ is naturally engaged in shaping synaptic activity by imposing a refractory period after a burst of cooperative synaptic activity.

\section{References}

Abbott LF, Regehr WG (2004) Synaptic computation. Nature 431:796-803.

Abrahamsson T, Gustafsson B, Hanse E (2005) Synaptic fatigue at the naive perforant path-dentate granule cell synapse in the rat. J Physiol 569: $737-750$.

Andersen P (1960) Interhippocampal impulses. II. Apical dendritic activation of CAI neurons. Acta Physiol Scand 48:178-208.

Andersson M, Blomstrand F, Hanse E (2007) Astrocytes play a critical role in transient heterosynaptic depression in the rat hippocampal CA1 region. J Physiol 585:843-852.

Araque A, Parpura V, Sanzgiri RP, Haydon PG (1999) Tripartite synapses: glia, the unacknowledged partner. Trends Neurosci 22:208-215.

Arnth-Jensen N, Jabaudon D, Scanziani M (2002) Cooperation between independent hippocampal synapses is controlled by glutamate uptake. Nat Neurosci 5:325-331.

Billups B, Graham BP, Wong AY, Forsythe ID (2005) Unmasking group III metabotropic glutamate autoreceptor function at excitatory synapses in the rat CNS. J Physiol 565:885-896.

Blomstrand F, Venance L, Sirén AL, Ezan P, Hanse E, Glowinski J, Ehrenreich H, Giaume C (2004) Endothelins regulate astrocyte gap junctions in rat hippocampal slices. Eur J Neurosci 19:1005-1015.

Bowser DN, Khakh BS (2007) Two forms of single-vesicle astrocyte exocytosis imaged with total internal reflection fluorescence microscopy. Proc Natl Acad Sci U S A 104:4212-4217.

Bushong EA, Martone ME, Ellisman MH (2004) Maturation of astrocyte morphology and the establishment of astrocyte domains during postnatal hippocampal development. Int J Dev Neurosci 22:73-86.

Diamond JS (2005) Deriving the glutamate clearance time course from transporter currents in CA1 hippocampal astrocytes: transmitter uptake gets faster during development. J Neurosci 25:2906-2916.

Duan S, Anderson CM, Keung EC, Chen Y, Chen Y, Swanson RA (2003) $\mathrm{P} 2 \mathrm{X} 7$ receptor-mediated release of excitatory amino acids from astrocytes. J Neurosci 23:1320-1328.

Fonnum F, Johnsen A, Hassel B (1997) Use of fluorocitrate and fluoroacetate in the study of brain metabolism. Glia 21:106-113.

Giaume C, Koulakoff A, Roux L, Holcman D, Rouach N (2010) Astroglial networks: a step further in neuroglial and gliovascular interactions. Nat Rev Neurosci 11:87-99.

Gustafsson B, Asztely F, Hanse E, Wigström H (1989) Onset characteristics of long-term potentiation in the guinea-pig hippocampal CA1 region in vitro. Eur J Neurosci 1:382-394.

Henneberger C, Papouin T, Oliet SH, Rusakov DA (2010) Long-term potentiation depends on release of D-serine from astrocytes. Nature 463:232-236

Huerta PT, Lisman JE (1996) Synaptic plasticity during the cholinergic theta-frequency oscillation in vitro. Hippocampus 6:58-61.

Jourdain P, Bergersen LH, Bhaukaurally K, Bezzi P, Santello M, Domercq M, Matute C, Tonello F, Gundersen V, Volterra A (2007) Glutamate exocytosis from astrocytes controls synaptic strength. Nat Neurosci 10: 331-339.

Konietzko U, Müller CM (1994) Astrocytic dye coupling in rat hippocampus: topography, developmental onset, and modulation by protein kinase C. Hippocampus 4:297-306.

Larson J, Wong D, Lynch G (1986) Patterned stimulation at the theta fre- quency is optimal for the induction of hippocampal long-term potentiation. Brain Res 368:347-350.

Li D, Ropert N, Koulakoff A, Giaume C, Oheim M (2008) Lysosomes are the major vesicular compartment undergoing $\mathrm{Ca}^{2+}$-regulated exocytosis from cortical astrocytes. J Neurosci 28:7648-7658.

Lisman J, Buzsáki G (2008) A neural coding scheme formed by the combined function of gamma and theta oscillations. Schizophr Bull 34:974980.

Liu QS, Xu Q, Kang J, Nedergaard M (2004) Astrocyte activation of presynaptic metabotropic glutamate receptors modulates hippocampal inhibitory synaptic transmission. Neuron Glia Biol 1:307-316.

Marchaland J, Calì C, Voglmaier SM, Li H, Regazzi R, Edwards RH, Bezzi P (2008) Fast subplasma membrane $\mathrm{Ca}^{2+}$ transients control exo-endocytosis of synaptic-like microvesicles in astrocytes. J Neurosci 28:9122-9132.

Neher E, Sakaba T (2008) Multiple roles of calcium ions in the regulation of neurotransmitter release. Neuron 59:861-872.

Newman EA (2003) New roles for astrocytes: regulation of synaptic transmission. Trends Neurosci 26:536-542.

Pascual O, Casper KB, Kubera C, Zhang J, Revilla-Sanchez R, Sul JY, Takano H, Moss SJ, McCarthy K, Haydon PG (2005) Astrocytic purinergic signaling coordinates synaptic networks. Science 310:113-116.

Perea G, Navarrete M, Araque A (2009) Tripartite synapses: astrocytes process and control synaptic information. Trends Neurosci 32:421-431.

Perez-Gonzalez AP, Albrecht D, Blasi J, Llobet A (2008) Schwann cells modulate short-term plasticity of cholinergic autaptic synapses. J Physiol 586:4675-4691.

Piet R, Vargová L, Syková E, Poulain DA, Oliet SH (2004) Physiological contribution of the astrocytic environment of neurons to intersynaptic crosstalk. Proc Natl Acad Sci U S A 101:2151-2155.

Robitaille R (1998) Modulation of synaptic efficacy and synaptic depression by glial cells at the frog neuromuscular junction. Neuron 21:847-855.

Sakaba T, Schneggenburger R, Neher E (2002) Estimation of quantal parameters at the calyx of Held synapse. Neurosci Res 44:343-356.

Santello M, Volterra A (2009) Synaptic modulation by astrocytes via Ca2+dependent glutamate release. Neuroscience 158:253-259.

Schneggenburger R, Sakaba T, Neher E (2002) Vesicle pools and short-term synaptic depression: lessons from a large synapse. Trends Neurosci 25: 206-212.

Schools GP, Zhou M, Kimelberg HK (2006) Development of gap junctions in hippocampal astrocytes: evidence that whole cell electrophysiological phenotype is an intrinsic property of the individual cell. J Neurophysiol 96:1383-1392.

Serrano A, Haddjeri N, Lacaille JC, Robitaille R (2006) GABAergic network activation of glial cells underlies hippocampal heterosynaptic depression. J Neurosci 26:5370-5382.

Verkhratsky A, Orkand RK, Kettenmann H (1998) Glial calcium: homeostasis and signaling function. Physiol Rev 78:99-141.

Witcher MR, Kirov SA, Harris KM (2007) Plasticity of perisynaptic astroglia during synaptogenesis in the mature rat hippocampus. Glia 55:13-23.

Zhang JM, Wang HK, Ye CQ, Ge W, Chen Y, Jiang ZL, Wu CP, Poo MM, Duan S (2003) ATP released by astrocytes mediates glutamatergic activity-dependent heterosynaptic suppression. Neuron 40:971-982.

Zhang Z, Chen G, Zhou W, Song A, Xu T, Luo Q, Wang W, Gu XS, Duan S (2007) Regulated ATP release from astrocytes through lysosome exocytosis. Nat Cell Biol 9:945-953.

Zhou M, Schools GP, Kimelberg HK (2006) Development of GLAST(+) astrocytes and $\mathrm{NG2}(+)$ glia in rat hippocampus CA1: mature astrocytes are electrophysiologically passive. J Neurophysiol 95:134-143.

Zucker RS, Regehr WG (2002) Short-term synaptic plasticity. Annu Rev Physiol 64:355-405. 